Voix et Images

voixetimages

\title{
Bibliographie D’Élise Turcotte
}

\section{Camille Allaire et Jonathan Lamy}

Volume 31, numéro 3 (93), printemps 2006

Élise Turcotte

URI : https://id.erudit.org/iderudit/013240ar

DOI : https://doi.org/10.7202/013240ar

Aller au sommaire du numéro

\section{Éditeur(s)}

Université du Québec à Montréal

\section{ISSN}

0318-9201 (imprimé)

1705-933X (numérique)

Découvrir la revue

\section{Citer ce document}

Allaire, C. \& Lamy, J. (2006). Bibliographie D’Élise Turcotte. Voix et Images, 31(3), 75-84. https://doi.org/10.7202/013240ar d'utilisation que vous pouvez consulter en ligne.

https://apropos.erudit.org/fr/usagers/politique-dutilisation/ 


\title{
B IB L I O G R A P H IE D’ÉLISE T U R COTTE
}

\author{
CAMILLE ALLAIRE \\ Université du Québec à Montréal \\ avec la collaboration de JONATHAN LAMY \\ Université du Québec à Montréal
}

\section{E U V R E S}

\section{1. Rom a n s}

+ Le bruit des choses vivantes, Montréal, Leméac, 1991, 227 p. ; Arles, Actes Sud, coll. «Babel», 1998, $244 \mathrm{p}$.

+ Lî́le de la Merci, Montréal, Leméac, 1997, 202 p. ; Montréal, Bibliothèque québécoise, 2001, $211 \mathrm{p}$.

+ La maison étrangère, Montréal, Leméac, 2002, 221 p.

\section{2. Nouvelles}

+ La mer à boire, Montréal, Éditions de la Lune occidentale, 1980, 24 p.

+ Caravane, Montréal, Leméac, 1994, 167 p. ; Saint-Laurent, Bibliothèque québécoise, 2004, $141 \mathrm{p}$.

\section{I . 3. Pó é si e}

+ Dans le delta de la nuit, Trois-Rivières, Écrits des Forges, 1982, 61 p.

+ Navires de guerre, Trois-Rivières, Écrits des Forges, 1984, 61 p.

+ La voix de Carla, Montréal, VLB éditeur, 1987, 97 p. ; Montréal, Leméac, coll. «Poésie», 1999, $93 \mathrm{p}$.

+ La terre est ici, Montréal, VLB éditeur, 1989, 106 p. ; Montréal, Éditions du Noroît, 2003, 105 p.

+ Deux ou trois feux, poèmes d'Élise Turcotte et photographies de Jocelyne Alloucherie, Montréal, Dazibao, Centre de photographies actuelles, 1997, 69 p.

+ Sombre ménagerie, Montréal, Éditions du Noroît, 2002, 71 p.

+ Voyages autour de mon lit, Montréal, La courte échelle, coll. «Poésie», 2002, 35 p.

+ Diligence: de Pike River à Beebe Plain, Longueuil, Les Petits villages, 2004. 28 p. Édition limitée à 200 exemplaires numérotés.

+ Piano mélancolique, Montréal, Éditions du Noroît, 2005, 88 p.

\section{I . 4. Rom ans je un esse}

+ Les cahiers d'Annette, Montréal, La courte échelle, coll. «Premier roman», 1998, 58 p.

+ La leçon d'Annette, Montréal, La courte échelle, coll. "Premier roman», 1999, 61 p.

+ Annette et le vol de nuit, Montréal, La courte échelle, coll. «Premier roman», 2000, 60 p. 
+ Guillaume Rioux, le poisson orphelin, avec Marc Mongeau, Montréal, La courte échelle, coll. «Il était une fois», 2001, 24 p.

+ Mes animaux, Montréal, La courte échelle, coll. «Puce», 2001, 16 p.

+ Ma famille, Montréal, La courte échelle, coll. «Puce», 2001, 16 p.

+ Ma maison, Montréal, La courte échelle, coll. «Puce», 2001, 16 p.

+ Mes douceurs, Montréal, La courte échelle, coll. «Puce», 2001, 16 p.

+ C'est bizarre, Montréal, La courte échelle, coll. «Puce», 2003, 16 p.

+ C'est sale, Montréal, La courte échelle, coll. «Puce», 2003, 16 p.

\section{I.5. Publications dans les périodiques}

+ «Absence et suite», La Nouvelle Barre du jour, no 70, octobre 1978, p. 5-17.

+ «Ça dit l'image», La Nouvelle Barre du jour, n 81, septembre 1979, p. 29-42.

+ «Langue de chat», La Nouvelle Barre du jour, nos 92-93, juin 1980, p. 237-243.

+ «Du désert rouge», Mœbius, no 14, printemps 1982, p. 7-12.

+ "Des rendez-vous manqués sous la voilette noire du temps», La Nouvelle Barre du jour, nº 124, mars 1983, p. 57-62.

+ «La chambre», Lèvres urbaines, n 2, septembre 1983, p. 7.

+ "Un autre geste à côté de la mémoire», La Nouvelle Barre du jour, no 140, juin 1984, p. 118.

+ «Petites planètes et voitures», Estuaire, nos 32-33, automne 1984, p. 132-133.

+ «Fragments de mémoire» (extraits du mémoire déposé à l'Université du Québec à Montréal), en collaboration avec Danielle Laurin, Mœbius, n²2, été 1984, p. 23-30.

+ "Extraits du livre de bord ou la discontinuité», Question de culture, n 8, avril 1985, p. 19-26.

+ «Le roman et la voix de Carla», Estuaire, n 37, automne 1985, p. 31-35.

+ «Le grain et la géographie», La Nouvelle Barre du jour, n 181, septembre 1986, p. 5-15.

+ «Les paysages», Travers, nos 31-32, juin 1987, p. 19-23.

+ «Les paysages», Estuaire, n 46, automne 1987, p. 41-42.

+ «Le sablier», Lèvres urbaines, n 17 , automne 1987, non paginé.

+ «Les paysages», Estuaire, n 50, automne 1988, p. 97-98.

+ «Navires de guerre (extraits)», traduction de Jo-Ann Elder, Ellipse, n 39, 1988, p. 44-45.

+ «Les lettres entières», en collaboration avec Louise Desjardins, La Nouvelle Barre du jour, avril 1989, p. 11-27.

+ «Trois jours», Estuaire, n 58, décembre 1990, p. 25-27.

+ «L'avenir de la solitude», Trois, vol. 6, nos 2-3, hiver-printemps 1991, p. 226-228.

+ «Ici», Estuaire, nos 65-66, automne 1992, p. 62-64.

+ «Les petites lumières», Arcade, no 25, automne 1992, p. 15-16.

+ «Road movie: une histoire vraie», en collaboration avec Carole David, Estuaire, n 68, printemps 1993, p. 27-40.

+ «Conversation», Stop, no 131, été 1993, p. 59-62.

+ «Tout est donné (extraits)», Estuaire, nº 72, janvier 1994, p. 49-53.

+ «Tout est donné», Possibles, vol. 18, nº 1, hiver 1994 p. 133-136.

+ "Vintage Clothes», traduction de Sheila Fischman, Matrix, n 46, 1995, p. 37-38.

+ «Joyce Carol Oates lue par Élise Turcotte», Voir, cahier «Livres», vol. 1, n 9, mars 1995, p. 8.

+ «Je ne t'ai pas reconnu», Estuaire, nos 80-81, mars 1996, p. 256.

+ «Friperie», Semestrial Publicacion de literatura, no 7, mars-avril 1997, p. 47-55.

+ «Jamaïque», Estuaire, nº 88, septembre 1997, p. 44. 
+ «Le vierge exquis», Estuaire, n ${ }^{9}$ 3, septembre 1998, p. 101.

+ «Deux ou trois feux (extraits)», Europe, no 839, mars 1999, p. 211-213.

+ «Vision d'un geste», Art Le Sabord, n 53, automne 1999, p. 12.

+ «La mer à boire», Mœbius, no 100, 2003, p. 11-17.

+ «Sombre ménagerie (extraits)», L'Arbre à paroles, janvier-février-mars 2003, p. 40.

+ «Piano mélancolique (extraits), Jet d'encre, n 3, printemps 2003, p. 59-62.

+ «Sans titre», traduction d'un poème par Silvia Eugenia Castillero, Luvina, n 33, décembre 2003, p. 7.

+ «Hôtel Éternité», Estuaire, n 120, septembre 2004, p. 75-79.

+ «La musique te soulève», Rampike, vol. 14, nº 1, numéro spécial, 2005, p. 5.

+ «Melancholy piano», traduction d'Adrienne Rich, The New Review of Literature, vol. 2, n 2, avril 2005, p. 98-99.

+ «Ā la manière noire. Autoportrait», Lettres québécoises, nº 120, hiver 2005, p. 5.

\section{I.6. Publications dans des anthologies ou ouvrages collectifs}

+ «Les chambres d'abstraction», Endre Farkas, Lucien Francoeur, Ken Norris et Ruth Taylor (dir.), Montréal Now!, Sainte-Anne-de-Bellevue, 1984, non paginé.

+ «Le portrait de nous devant la vitrine», Qui a peur de?, Montréal, VLB éditeur, 1987, p. 101106.

+ «Nature morte», Louise Warren (dir.), La poésie mémoire de l'art. Anthologie, Trois-Rivières, Éditions Art Le Sabord, 2003, p. 64.

+ "Québec comme bondissement de comète», Roger Chamberland (dir.) Anthologie de poésie québécoise, Sainte-Foy, Les Presses de l'Université Laval, 2002, p. 138-139.

+ «Vision de ma chambre pour Hildegarde», Marc Séguin et Benoît Villeneuve (textes littéraires sous la direction de Jean-François Poupart), Dialogue dans l'espace, Saint-Laurent, ERPI, 2002, p. 61.

+ Nicole Brossard et Lisette Girouard (dir.), Anthologie de la poésie des femmes au Québec des origines à nos jours, Montréal, Les Éditions du remue-ménage, 2003, p. 371-374.

+ Titti Follieri (dir.), Antologia della poesia contemporanea del Québec, Milan, Crocetti Editore, 1998, p. 120-127.

+ Raymonde April et Élise Turcotte, «Deux petites marches», Denise Desautels et Marcel Labine (dir.), Installations, fictions, Éditions La Nouvelle Barre du jour, nos 89-90, 1986, non paginé.

+ Louise Blouin (dir.), Des mots pour rêver. Anthologie de poésie québécoise, Montréal/TroisRivières, Éditions Pierre Tisseyre/Écrits des Forges, coll. «Conquêtes», 1990, p. 157-160.

+ Louise Desjardins et Élise Turcotte, La catastrophe, Éditions La Nouvelle Barre du jour, no 67 , $1985,45 \mathrm{p}$.

+ Louise Blouin et Bernard Pozier (dir.), Poetas de Quebec. Breve antologia, Trois-Rivières/ Mexico, Universidad Nacional Autonoma de Mexico/Écrits des Forges/Editorial Aldus, Mexico, 1996, p. 309-310.

+ Louise Blouin et Bernard Pozier (dir.), Poètes des Écrits des Forges. Petite anthologie de la poésie québécoise, Montréal, Bibliothèque québécoise, 2003, p. 136-140.

+ Louise Blouin et Bernard Pozier (dir.), Poètes québécois. Anthologie, Trois-Rivières, Écrits des Forges, 1996, p. 217-218. 


\section{TRADUCTION DES EUURES D'ÉLISE TURCOTTE}

+ The Sound of Living Things [Le bruit des choses vivantes], traduction de Sheila Fischman, Toronto, Coach House Press, 1993, 153 p; Toronto, Cormorant Books, 2003, 141 p.

+ El soroll de les coses vives [Le bruit des choses vivantes], traduction de Lourdes Bigorra, Barcelone, Edicions de la Magrana, 2001, 193 p.

+ My Animals [Mes animaux], Montréal, La courte échelle, 2001, 16 p.

+ My Family [Ma famille], Montréal, La courte échelle, 2001, 16 p.

+ My Home [Ma maison], Montréal, La courte échelle, 2001, 16 p.

+ My Cuddly Toys [Mes douceurs], Montréal, La courte échelle, 2001, 16 p.

+ Navios de guerra/Navires de guerre, traduction de Luis Armenta Malpica et Gabriel Martin, édition bilingue, Trois-Rivières/Tlaquepaque (Mexique), Les Écrits des Forges/Mantis Editores, 2002, $109 \mathrm{p}$.

+ The Body's Place [L'île de la Merci], traduction de Sheila Fischman, Toronto, Cormorant Books, 2003, $151 \mathrm{p}$.

+ En el delta de la noche [Dans le delta de la nuit], traduction de Gabriel Martin et Luis Armenta Malpica, édition bilingue, Trois-Rivières/Tlaquepaque (Mexique), Écrits des Forges/Mantis Editores, 2003, 105 p.

+ The Alien House [La maison étrangère], traduction de Sheila Fischman, Toronto, Cormorant Books, 2004, 243 p.

\section{PRIX ET D IS TINCTIONS}

+ Prix Émile-Nelligan 1987 pour La voix de Carla.

+ Prix Émile-Nelligan 1989 pour La terre est ici.

+ Prix Louis-Hémon 1991 pour Le bruit des choses vivantes.

+ Bourse Gabrielle-Roy 1995.

+ Grand Prix du Festival international de la poésie 2002 pour Sombre ménagerie.

+ Prix de poésie des Terrasses Saint-Sulpice de la revue Estuaire 2002 pour Sombre ménagerie.

+ Prix littéraire du Gouverneur général 2003 pour La maison étrangère.

\section{RÉCEPTION CRITIQUE ET ÉTUDES}

\section{IV.1. Articles de fond et chapitres de livres}

+ BROCHU, André, "Ceux de demain", Tableaux du poème. La poésie québécoise des années quatre-vingt, Montréal, XYZ éditeur, coll. «Documents», 1994, p. 22-23.

+ CARON, Valérie, «Le bruit des choses vivantes et Tableaux [de D. Kimm] : voix et représentations inédites de la maternité dans la littérature québécoise », Voix et Images, n 82 , automne 2002, p. 126-141.

+ LAROCHELLE, Corinne, «Lire l'image: Le bruit des choses vivantes d'Élise Turcotte», Voix et Images, nº 69, printemps 1998, p. 544-557.

+ LÉTOURNEAU, Sophie, «Le corps froid de l'amour», Spirale, nº 198, septembre-octobre 2004, p. 18-19.

+ NAREAU, Michel, «Le Nord indéterminé et intertextuel dans les œuvres de Lise Tremblay, Élise Turcotte et Pierre Gobeil», BOUCHARD, Joë, CHARTIER, Daniel et Amélie NADEAU (dir.), Problématiques de l'imaginaire du Nord en littérature, cinéma et arts visuels, Université du Québec à Montréal, Département d'études littéraires, coll. «Figura. Centre de recherche sur le texte et l'imaginaire», 2004, p. 41-58. 
+ NEPVEU, Pierre, «Les choses vivantes d'Élise Turcotte», Lectures des lieux, Montréal, Boréal, coll. «Papiers collés», 2004, p. 209-217.

+ SAINT-MARTIN, Lori, «Le métaféminisme et la nouvelle prose féminine au Québec », Voix et Images, n 52, automne 1992, p. 78-88.

+ SAINT-MARTIN, Lori, «Le corps et la fiction à réinventer», Recherches féministes, vol. 7, nº 2 , 1994, p. 115-134.

+ SAINT-MARTIN, Lori, «Le bruit des choses vivantes d'Élise Turcotte: “Maman, lis-moi ce que j'ai écrit" ", Le nom de la mère: mères, filles et écriture dans la littérature québécoise au féminin, Montréal, Nota bene, coll. «Essais critiques», 1999, p. 283-295.

+ VAUDRY, Catherine, «S'écrire corps et âme. La quête des traces et des souvenirs dans La maison étrangère d'Élise Turcotte», Postures, nº 6, 2004, p. 91-103.

\section{V. 2. Mémoires et thè se s}

+ CARON, Valérie, «Voix et représentations inédites de la maternité dans la littérature québécoise au féminin, étude, suivie de La terre retrouvée, récit», mémoire de maîtrise, Sherbrooke, Université de Sherbrooke, 2003, $124 \mathrm{f}$.

+ CLENDINNING, Sherri Lee, «La manifestation littéraire du discours maternel: L'obéissance de Suzanne Jacob et Le bruit des choses vivantes d'Élise Turcotte», mémoire de maîtrise, Ottawa, Carleton University, 1994, 98 f.

+ GERMAIN, Brigitte, "Configurations du sujet féminin dans Le bruit des choses vivantes d'Élise Turcotte», mémoire de maîtrise, Montréal, Université du Québec à Montréal, 2000, 135 f.

+ LAROCHELLE, Corinne, «La tentation du romanesque: analyse sociopoétique du roman Le bruit des choses vivantes d'Élise Turcotte", mémoire de maîtrise, Montréal, Université du Québec à Montréal, 1999, $130 \mathrm{f}$.

+ MADDOX, Kelly-Anne Madeline, «Le roman québécois des années 1990 : éléments fondamentaux de la problématique identitaire», thèse de doctorat, Halifax, Dalhousie University, 2004, $278 \mathrm{f}$.

+ POIRIER, Maryse, "Analyse sémiotique de la construction du personnage dans l'œuvre d'Élise Turcotte», mémoire de maîtrise, Sainte-Foy, Université Laval, 1998, 147 f.

+ ROSSI, Claudia Y., «L'enfance et l'adolescence chez Élise Turcotte», mémoire de maîtrise, Halifax, Dalhousie University, 2001, 165 f.

\section{IV.3. Entretiens et portraits}

+ AMYOT, Linda, «Élise Turcotte: Une géographie du monde», Nuit blanche, n 90, printemps 2003, p. 8-13.

+ CLOUTIER, Raymond «Élise Turcotte : une musique mélancolique», entretien radiophonique diffusé sur les ondes de Radio-Canada, 4 septembre 2005.

+ CORRIVEAU, Hugues, «Élise Turcotte et la vie soluble», Lettres québécoises, n 120, hiver 2005, p. 9-10.

+ GAUDET, Gérald, «L'orage des meilleures passions», Voix d'écrivains, Montréal, Québec Amérique, 1985, p. 275-279.

+ LAURIN, Danielle, «Entretien sur fond de musique noire, terrible et lancinante», Inter. Magazine de l'Université du Québec à Montréal, printemps 2004, p. 11-14.

+ MOLIN-VASSEUR, Annie, "Une mémoire grand-angulaire», Arcade, n 41, automne 1997, p. 65-76. 
+ PÉAN, Stanley, "Élise Turcotte. Se perdre dans son propre décor », Le Libraire, n 17, hiver 2002, p. 13.

+ SERGENT, Julie, «Sur les traces d'Élise Turcotte», Lettres québécoises, n 120, hiver 2005, p. 6-8.

+ VIGNEAULT, Alexandre, "Portions de réalités», Le Quartier libre, vol. 1, nº 15, 25 avril 1994, p. 19.

\section{IV.4. Comptes rendus et articles de presse}

\section{IV.4.1. Dans le delta de la nuit}

+ BEAULIEU, Michel, «Élise Turcotte et la quête d'intériorité», Livres d'ici, vol. 7, n 50, septembre 1982, non paginé.

+ BEAUSOLEIL, Claude, «La marche des mots», Le Devoir, 15 avril 1983, p. 24.

+ BEAUSOLEIL, Claude, "Lectures d'errances actuelles», La Nouvelle Barre du jour, nº 129, septembre 1983, p. 103-104.

+ BOUCHARD, Christian, «Estuaire a reçu. Dans le delta de la nuit», Estuaire, n 27, printemps, 1983, p. 89.

+ CORRIVEAU, Hugues, "Dans le delta de la nuit», Livres et auteurs québécois, 1982, p. 141-142.

+ DIONNE, André, "Dans le delta de la nuit», Nos livres, vol. 15, juillet-août 1983, p. 55-56.

+ DORION, Hélène, "Dans le delta de la nuit», Québec français, n 48, décembre 1982, p. 6.

+ DUPRÉ, Louise, «Deux accents de mémoire», Spirale, nº 30, décembre 1982, p. 11.

+ FORTIER, Éric, "Dans le delta de la nuit et Navires de guerre», Aurélien Boivin (dir.),

Dictionnaire des œuvres littéraires du Québec, t. VII, 1981-1985, Saint-Laurent, Fides, 2003, p. 210-212.

+ LAWALL, Sarah, "Dans le delta de la nuit», The French Review, vol. 58, n 3, février 1985, p. 501-503.

+ MARTIN, Raymond, «Dans le delta de la nuit», Mœbius, nº 6, hiver 1983, p. 88.

\section{IV.4.2. Navires de guerre}

+ BEAULIEU, Michel, «Navires de guerre», Livre d'ici, janvier 1985, p. 10.

+ BEAUSOLEIL, Claude, «Voguer à la lisière de l'imaginaire», Liaison St-Louis, nº 8, 10 avril 1985, p. 11.

+ BROCHU, André, "Des fous et des autres», Voix et Images, vol. 10, n 3, printemps 1985, p. 179-187.

+ CHAMBERLAND, Roger, «Navires de guerre», Québec français, n 58, mars 1985, p. 10.

+ CHASSAY, Jean-François, «Écrire, dit-elle», Spirale, no 49, février 1985, p. 7.

+ CHOUINARD, Lucie, "Navires de guerre», Nuit blanche, n²0, octobre-novembre 1985, p. 19.

+ DUPRÉ, Louise, «Poetry returns to love», Ellipse, 1988, p. 13.

+ FOURNIER, Danielle, «Navires de guerre», Arcade, no 10, octobre 1985, p. 64-65.

+ GAUDET, Gérald, «L'orage des meilleures passions», Le Devoir, 29 décembre 1984, p. 17 et 21.

+ GUÉNETTE, Daniel, «Navires de guerre», Nos livres, mars 1985, p. 39-40.

+ MARQUIS, André, "Ah! L'amour, la poésie», Lettres québécoises, n 38, été 1985, p. 33-34.

+ MONTPLAISIR, Liane, "Navires de guerre», Art Le Sabord, n 5, décembre 1984, p. 20.

+ PAQUIN, Jacques, «Navires de guerre», Estuaire, n 34, hiver 1985, p. 80.

+ ROY-GUÉRIN, Michelle, «Deux nouveaux titres aux Écrits des Forges», Le Nouvelliste, 24 novembre 1984, p. 13.

+ ROYER, Jean, «La poésie qui se fréquente», Le Devoir, 15 décembre 1984, p. 27. 
+ SAINT-PIERRE, Jacques, "Archives distraites. Navires de guerre. Si l'herbe pour toujours", Mobius, no 23, 1984, p. 90-91.

+ YERGEAU, Robert, «Mêler les styles... et autres errances », University of Toronto Quaterly, vol. 54, nº 4, été 1985, p. 369.

\section{IV.4.3. La voix de Carla}

+ D’ALFONSO, Antonio, "La voix de Carla», Estuaire, n 46, automne 1987, p. 89-90.

+ GIGUÈRE, Richard, «La tentation du romanesque», Lettres québécoises, n 47, automne 1987, p. 37-39.

+ TOUPIN, Gilles, «Des notes pour ne pas passer à côté de la vie», La Presse, 30 mai 1987, p. E2.

\section{IV.4.4. La terre est ici}

+ CÔTÉ, Lucie, «La terre est ici», La Presse, 31 mars 1990, p. K4.

+ FORTIN, Marie-Claude, «Une minute de silence», Voir, 14 décembre 1989, p. 29.

+ LATENDRESSE, Claude, "La terre est ici», Estuaire, n 57, automne 1990, p. 69-70.

+ MARCOTTE, Hélène, "La terre est ici ", Nuit blanche, no 40, juillet-août 1990, p. 29.

+ MARQUIS, André, "Odes à l'amour», Lettres québécoises, n 59, automne 1990, p. 37-38.

+ TOUPIN, Gilles, «La terre d'Élise Turcotte», La Presse, 20 janvier 1990, p. K3.

\section{IV.4.5. Le bruit des choses vivantes}

+ [Anonyme], «La bibliothèque de l'été. Lire en vacances...», La Presse, 16 juin 1990, p. K1.

+ BERGERON, Lise, "Le bruit des choses vivantes », Art le Sabord, n 30, hiver 1992, p. 38-39.

+ BERTIN, Raymond, "La mémoire et le rêve», Guide Mont-Royal, 23 octobre 1991.

+ BOIS Ann, "Le bruit des choses vivantes», The Gazette, 28 décembre 1991, p. H9.

+ BYRNE, Kathleen, "The Sound of Living Things», The Globe and Mail, 12 février 1994, p. C18.

+ CAMPEAU, Francine, "Le bruit des choses vivantes», Mœbius, n 52, printemps 1992, p. 160-162.

+ CARCASONNE, Manuel, "Québec. Le pays retrouvé», Le Point, n 1383, 20 mars 1999, p. 107.

+ CHAMBERLAND, Roger, "Le bruit des choses vivantes", Québec français, n 84, hiver 1992, p. 16.

+ CNOCKAERT, Véronique, «Témoignage», Spirale, n 111, décembre 1991, p. 16.

+ CORNELLIER, Louis, "Chorégraphie d'êtres déboussolés», Le Devoir, 9 novembre 1991, p. 32.

+ CORRIVEAU, Hugues, «La vérité des choses réelles», Lettres québécoises, nº 67, automne 1992, p. 9-10.

+ CÔTÉ, Lucie, «Élise Turcotte : une belle voix s'élève», La Presse, 13 octobre 1991, p. C1.

+ CÔTÉ, Lucie, «Un premier roman", La Presse, 24 janvier 1992, p. C1.

+ FORTIN, Marie-Claude, «Le grand cahier», Voir, 10 octobre 1991, p. 24.

+ FORTIN, Marie-Claude, "Élise Turcotte en quête du sens de la vie», Page des libraires, n 56, février 1994, p. 41.

+ GILLILAND, Gail, «A Mother's Meditation», The New York Times, 2 janvier 1994, p. BR14.

+ GIRARD, Marie-Claire, «Un livre magnifique et bouleversant», Le Droit, 12 octobre 1991, p. A8.

+ LAMONTAGNE, Marie-Andrée, «Le petit bout de la lorgnette», Montréal, avril 1992, p. 41.

+ LOVE, Barbara, "Le bruit des choses vivantes», Library Journal, $1^{\text {er }}$ novembre 1993.

+ MANION, Eileen, "The Right Words for it», Books in Canada, vol. 22, n 9, décembre 1993, p. $44-45$. 
+ MARCOTTE, Gilles, «Un moment de grâce», L'Actualité, $1^{\text {er }}$ mars 1992, p. 75.

+ NACHTSHEIM, M. H., "Le bruit des choses vivantes», Choice, vol. 31, nº 8, avril 1994, p. 1298.

+ POULIN, Andrée, "De la famille et des mille manières d'en parler", Lettres québécoises, n 64 , hiver 1991-1992, p. 22-23.

+ POULIN, Andrée, "Comme un petit chat triste», Le Droit, 2 avril 1994, p. A6.

+ SHERIFF, Candace, "Le bruit des choses vivantes», The Vancouver Sun, 19 mars 1994, p. D18.

\section{IV.4.6. Caravane}

+ ALLARD, Jacques, "Trente-cinq ans, deux enfants, des copines et des amants», Le Devoir, 26 février 1994, p. D5.

+ BÉLANGER, Reine, "Caravane», Nuit blanche, n 57, octobre-novembre 1994, p. 34-35.

+ BERGERON, Lise, "Turcotte Caravane», Art Le Sabord, no 38, automne 1994, p. 39.

+ CAYOUETTE, Pierre, «Porter le chagrin des départs», Le Devoir, 26 février 1994, p. D1-D2.

+ CHAMBERLAND, Roger, "Caravane», Québec français, n 94, été 1994, p. 11.

+ CHAREST, Remy, «Caravane», The Gazette, 19 mars 1994, p. I5.

+ FORTIN, Marie-Claude, «Terre humaine», Voir, cahier spécial, 3 mars 1994, p. 6.

+ HOMMEL, Christian, «Sans doute le meilleur recueil de nouvelles de l'année!», Impact Campus, 22 mars 1994, p. 21.

+ LEQUIN, Lucie, «Une image lui bloquait la vue», Voix et Images, n 57, printemps 1994, p. 649-654.

+ MARCOTTE, Gilles, "Quinze histoires d'Élise Turcotte», L'Actualité, vol. 19, nº 6, avril 1994, p. 73.

+ MARTEL, Réginald, «Histoires d'abandon et de géométrie amoureuse», La Presse, 13 février 1994, p. B7.

+ POTVIN, Claudine, «Le monde des hommes vu par Marie», Lettres québécoises, n 75, automne 1994, p. 38.

+ VOISARD, Anne-Marie, "Observer la vie au féminin, singulier », Le Soleil, 21 février 1994, p. B7.

\section{IV.4.7. L'île de la Merci}

+ [Anonyme], "L'île de la Merci», Le Libraire, novembre 2001, p. 45.

+ BIRON, Michel, «Deconstructing Marcel», Voix et Images, n 69, printemps 1998, p. 593-597.

+ BIRRELL, Heather, «No Body's Place for Love», Books in Canada, vol. 32, n 6, septembre 2003, p. 7.

+ CAYOUETTE, Pierre, «Écrire la dureté du monde», Le Devoir, 30 août 1997, p. D1.

+ CLEMENCE, Verne, "Translation Open Doors to Literature», Saskatoon Starphoenix, 12 juillet 2003, p. E4.

+ DONNELLY, Pat, «Giving Voice to Teen Angst», The Gazette, 26 juillet 2003, p. I3.

+ ERMEN, Don, «Lyrical Books Wrap You Like a Warm Blanket», Ottawa Sun, 27 juillet, 2003.

+ FORTIN, Marie-Claude, "L'écriture ou la vie», Lettres québécoises, hiver 1998, p. 16.

+ GUAY, Hervé, «Merci la mort», Le Prof, octobre 1997.

+ HANCOCK, Geoff, "Death and the Maiden», The Globe and Mail, 30 août 2003, p. D11.

+ LEBEL, Dominique, "Lîle de la Merci», La Terre de chez nous, 23 octobre 1997, p. 33.

+ MARTEL, Réginald, «Élise Turcotte: une œuvre forte», La Presse, 7 septembre 1997, p. B3.

+ MARCOTTE, Gilles, «Ah! Les mères... », L'Actualité, vol. 22, n 18, novembre 1997, p. 119.

+ NAVARRO, Pascale, «La vie devant soi», Voir, 4 septembre 1997, p. 39. 
+ NAVARRO, Pascale, «Pièces d'identité», Voir, cahier spécial, 11 septembre 1997, p. 4.

+ SERGENT, Julie, «Rencontres forcées», Le Devoir, 6 septembre 1997, p. D7.

+ SODERSTROM, Mary, «Lî́le de la Merci», Quill \& Quire, vol. 69, nº 5, 1r mai 2003.

+ VIGNEAULT, Alexandre, «Deux filles en rogne», La Presse, 14 octobre 2001, p. B2.

+ VIGNEAULT, Jean, «Un roman de portes qui claquent», Le Courrier de Saint-Hyacinthe, 3 septembre 1997, p. B5.

+ VOISARD, Anne-Marie, «Elle écrit au meurtre», Le Soleil, 30 août 1997, p. D8.

\section{IV.4.8. Les cahiers d'Annette}

+ DESROCHES, Gisèle, "Au-delà des histoires pour enfants», Le Devoir, 13 juin 1998, p. D6.

+ LACHANCE, Micheline, "Les cobayes d'Élise», L'Actualité, vol. 23, nº 7, 1 1er mai 1998, p. 90.

+ FRANCEUR, Martin, «L'été des jeunes lecteurs», Le Nouvelliste, 20 juin 1998, p. 6.

+ SARFATI, Sonia, "La même plume que pour les grands», La Presse, 24 mai 1998, p. B1-B2.

+ TREMBLAY, Carole, "Jeux d'enfants», Le Devoir, 9 mai 1998, p. D1-D2.

\section{IV.4.9. La leçon d'Annette}

+ [Anonyme], «Jeunesse: La leçon d'Annette», Voir, 25 mars 1999, p. 56.

+ LÉGARÉ, Isabelle, «Sale gosse!», Le Nouvelliste, 5 juin 1999, p. 7.

+ VIGNEAULT, Jean, «Le charmant, le parfait et le bizarre», Le Courrier de Saint-Hyacinthe, 17 février 1999, p. B5.

\section{IV.4.10. Annette et le vol de nuit}

+ VIGNEAULT, Jean, «Des détectives amateurs», Le Courrier de Saint-Hyacinthe, 30 août 2000, p. B4.

\section{IV.4.11. Guillaume Rioux, le poisson orphelin}

+ CHAREST, Remy, «L'imagination à la rescousse», Le Soleil, 25 novembre 2001, p. B3.

\section{IV.4.12. Voyage autour de mon lit}

+ CANTIN, David, «Dis-moi qui je suis», Le Devoir, 16 mars 2002, p. D2.

+ CORRIVEAU, Hugues, "Voyage autour de mon lit», Lettres québécoises, n 106, été 2002, p. 37.

+ SARFATI, Sonia, «La poésie dès l'adolescence», La Presse, 21 mars 2002, p. C2.

\section{IV.4.13. La maison étrangère}

+ BIRON, Michel, «Le symbolisme soft», Voix et Images, n 83, hiver 2003, p. 167-173.

+ BOURGAULT-CÔTÉ, Guillaume, «La mémoire, le corps et l'oubli», Le Soleil, 27 octobre 2002, p. B1-B2.

+ BROCHU, André, «La vie insatisfaite», Lettres québécoises, n 110, été 2003, p. 15-16.

+ GESSELL, Paul, "Glover, Turcotte Win for Fiction», The Gazette, 13 novembre 2003, p. A4.

+ LAPIERRE, Michel, «La disparition», Ici, 16 janvier 2003, p. 36.

+ LAURIN, Danielle, «Survivre au naufrage», Elle Québec, décembre 2002, p. 56.

+ MITCHELL, Elizabeth, «The Art of Daily Living», The Toronto Star, 9 janvier 2005, p. C18.

+ MORENCY, Catherine, «Les bestiaires obscurs», Le Devoir, 23 novembre 2002, p. F4.

+ NAVARRO, Pascale, «Élise Turcotte. Conte pour une femme seule», Voir, 31 octobre 2002, p. 16-17. 
+ ROTHMAN, Claire, "Cerebral Woman Learns from her Body», The Gazette, $1^{\text {er }}$ mai 2004, p. W4.

+ SARFATI, Sonia, "Visite guidée de La maison étrangère», La Presse, 27 octobre 2002, p. F3.

+ SODERSTROM, Mary, «The Alien House», Quill \& Quire, vol. 70, n 11, novembre 2004, p. 30.

+ TAYLOR, Kate, «Who's Afraid of Foreign Books? », The Globe and Mail, 11 mai 2005, p. R1.

+ TRATTFORD, Matthew, «La maison étrangère», The Dominion, vol. 2 n 7, mars 2005, p. 5.

\section{IV.4.14. Sombre ménagerie}

+ BEAUSOLEIL, Claude, «Pour Élise», La Presse, 22 septembre 2002, p. F3.

+ CANTIN, David, «Ne plus croire à la mort», Le Devoir, 13 juillet 2002, p. D3.

+ CANTIN, David, «Une ville en émoi», Le Devoir, 5 octobre 2002, p. F1.

+ DELAND, Monique, "Élise Turcotte, Sombre ménagerie», présentation des finalistes du prix de poésie Terrasses Saint-Sulpice de la revue Estuaire, Estuaire, n 115, 2003, p. 74-75.

+ LANDRY, Gabriel, «Sombre ménagerie», Voix et Images, n 83, hiver 2003, p. 187.

+ MALAVOY-RACINE, Tristan, «Voix parallèles», Voir, 26 septembre 2002, p. 40.

\section{IV.4.15. Piano mélancolique}

+ CORRIVEAU, Hugues, «Langueur du temps qui passe», Le Devoir, Montréal, 11 février 2006, p. F4.

+ JUTRAS, Benoît, «Sonate à la lune», Voir, 13 octobre 2005, p. 16.

\section{V.4.15. Autres}

+ BROCHU, André, «La folle quête de l'homme-mer», Mœbius, nº 100, hiver 2004, p. 7-9.

+ DE CORTANZE, Gérard, "D'une babel des lettres», Le Figaro littéraire, 19 mars 1999, p. 11.

+ FORTIN, Marie-Claude, "Élise Turcotte en quête du sens de la vie», Page des libraires, n 56 , février-mars 1999, p. 41.

+ LAPIERRE, Michel, «Les dragons fantômes d'Élise Turcotte», Ici, 22 juillet 2004, p. 38.

+ NAVARRO, Pascale, «Marc Rochette/Élise Turcotte», Voir, 30 mars 2000, p. 48. 